\title{
Preoperative Radiation Therapy is an Independent and Strong Risk Factor for Late Non-cancer Death after Esophagectomy
}

\author{
Takahiro MORI*, Shukichi MIYAZAKI, Go MIYATA, Ko SUGAWARA, \\ Ko ONODERA, Hirofumi ICHIKAWA, Takashi KAMEI, Hiroshi KIKUCHI, \\ Rikiya KANBA, Susumu SATOMI and Tatsuya TAKAHASHI ${ }^{1}$ \\ Division of Advanced Surgical Science and Technology, Tohoku University Graduate School of Medicine, \\ 1-1 Seiryo-cho, Aoba-ku, Sendai 980-8574, Japan \\ ${ }^{1}$ Division of Public Health and Preventive Medicine, Department of Diagnostic Information and \\ Socioenvironmental Medicine, Yamagata University School of Medicine, \\ 2-2-2 Iida-Nishi, Yamagata 990-9585, Japan
}

Received April 28, 2005; final version accepted July 6, 2005

\begin{abstract}
Objective: The aim of this study was to reveal the factors that influence the prognosis of esophageal cancer patients, especially from the other causes than esophageal cancer in the follow-up period after esophagectomy.

Method: All of 523 patients with esophageal carcinoma who underwent esophagectomy in a single institute between 1986 and 1999 were followed-up until the end of October 2003. Their prognoses were reviewed and compared by the presence of pre/post-operative radiation, pre/post-operative chemotherapy, surgical procedure, and pathological stages of tumors as well as preoperative general conditions by uni- and multivariate analyses.

Results: Univariate analysis revealed that sex (male against female) $(p=0.005)$, abnormality on ECG ( $p=0.012)$, and the presence of preoperative radiation $(p=0.016)$ significantly increased the incidence of nonesophageal cancer death; on the other hand, thoracoscopic approach decreased the incidence of non-esophageal cancer death. Multivariate analyses revealed that the presence of preoperative radiation significantly increased mortality due to non-esophageal cancer causes to a 3.70 hazard ratio (95\% CI [95\% Confidence Interval]; 1.33 10.61).

Conclusions: This study clearly showed the late effect of preoperative radiation for carcinoma of the esophagus on the postoperative prognosis, especially in terms of later mortality from causes other than esophageal carcinoma.
\end{abstract}

KEYWORDS: esophageal cancer, preoperative radiation, esophagectomy, cause of death

\section{Introduction}

Esophageal cancer is one of the most common malignant diseases in the world [17], and many efforts have been made to improve its prognosis that is relatively poor. Recently, it has been reported from many institutes that the combination of radiotherapy and chemotherapy with surgical operation for esophageal cancer has been successful in improving the prognosis; tri-modal therapy, which includes chemotherapy and radiotherapy as well as surgical treatment, is reported to be more effective against premature death from esophageal cancer [2, 4, 7, 10, 21, 23]. Hence, most medical institutes seem to prefer tri-modal therapy, especially initial radiochemotherapy followed by surgical treatment, so called neoadjuvant therapy, for esophageal cancer patients. However, initial radiation may render difficult the following surgical treatment and affect the prognosis of patients. In this paper, we reviewed 523 consecutive patients of thoracic esophageal carcinoma, including certain cervical esophageal carcinoma cases that require mediastinal manipulation because of mediastinal lymphnode metastasis or because tumor was located at the cervicothoracic junction, who underwent esophagectomy in a single institute, to examine the relationship between the prognosis and multi-modal therapy under controlled preoperative general conditions and the pathological stages by multivariate analysis. In the present study, it appeared likely that the presence of preoperative radiation therapy significantly increased the mortality from diseases other than esophageal cancer in the later follow-up period.

\section{Subjects and Methods}

Between January 1, 1986 and December 31, 1999, 523 consecutive patients with thoracic esophageal carcinoma or with cervical esophageal carcinoma-metastasized to a mediastinal lymphnode or located at the cervico-thoracic junction of the esophagus - underwent esophagectomy in Tohoku University Hospital at Sendai, Japan, excluding certain cases that did not require mediastinal manipulation and cases in which macroscopically curative resection could not be performed as listed in Table 1. They had been followed-up until as late as October 31, 2003, at Tohoku University Hospital and other affiliated hospitals. The prognoses were reviewed and analyzed in terms of preoperative conditions as well as pre- or post-operative adjuvant chemo/radiotherapies by univariate survival analyses as well as

* To whom corresponding should be addressed. E-mail: tamori-ths@umin.ac.jp 
Table 1. Characteristics of the study subjects.

\begin{tabular}{|c|c|}
\hline Preoperative examinations & \\
\hline ICG test $(<10 \%: \geqq 10 \%)$ & $285: 209$ (29cases, not determined) \\
\hline Electrocardiogram (cases with no abnormality found: cases with abnormalty found) & $331: 166$ (26cases, not determined) \\
\hline \multicolumn{2}{|l|}{ Preoperative adjuvant therapies } \\
\hline radiation only & 30 \\
\hline chemotherapy only & 21 \\
\hline concurrent radio-chemotherapy & 15 \\
\hline \multicolumn{2}{|l|}{ Postoperative adjuvant therapies } \\
\hline radiation only & 33 \\
\hline chemotherapy only & 68 \\
\hline concurrent radio-chemotherapy & 216 \\
\hline \multicolumn{2}{|l|}{ Pathological staging (JSED, ninth edition) } \\
\hline 0 & 36 \\
\hline I & 134 \\
\hline II & 48 \\
\hline III & 163 \\
\hline IVA or IVB & 134 \\
\hline
\end{tabular}

Table 2. Incidence of non-esophageal cancer death in parameters used in this study.

\begin{tabular}{|c|c|c|c|c|c|c|}
\hline factors examined & & $\begin{array}{c}\text { total number of } \\
\text { death from non-esophageal cancer }\end{array}$ & $\begin{array}{l}\text { total observed } \\
\text { person-years }\end{array}$ & incidence rate & $95 \%$ confidence intervals & Fisher's extact \\
\hline \multirow[t]{2}{*}{$\operatorname{sex}$} & male & 67 & 2166 & 0.031 & $0.024-0.039$ & $p=0.005$ \\
\hline & female & 5 & 471 & 0.011 & $0.004-0.025$ & \\
\hline \multirow[t]{2}{*}{ past history } & yes & 48 & 1618 & 0.03 & $0.022-0.039$ & $p=0.190$ \\
\hline & no & 24 & 1009 & 0.024 & $0.016-0.035$ & \\
\hline \multirow[t]{2}{*}{ ICG } & $>10$ & 31 & 1006 & 0.031 & $0.022-0.044$ & $p=0.158$ \\
\hline & normal & 36 & 1495 & 0.024 & $0.017-0.033$ & \\
\hline \multirow[t]{2}{*}{ ECG } & abnormal & 30 & 766 & 0.022 & $0.027-0.056$ & $p=0.012$ \\
\hline & normal & 39 & 1744 & 0.039 & $0.016-0.031$ & \\
\hline \multirow[t]{2}{*}{ preope. rad } & yes & 8 & 128 & 0.062 & $0.031-0.125$ & $p=0.016$ \\
\hline & no & 64 & 2509 & 0.026 & $0.020-0.033$ & \\
\hline \multirow[t]{2}{*}{ preope. chemo } & yes & 1 & 114 & 0.009 & $0.001-0.062$ & $p=0.109$ \\
\hline & no & 71 & 2523 & 0.028 & $0.022-0.036$ & \\
\hline \multirow{2}{*}{$\begin{array}{l}\text { surgical approach; } \\
\text { thoracotomy }\end{array}$} & yes & 59 & 2247 & 0.026 & $0.020-0.034$ & $p=0.216$ \\
\hline & no & 13 & 390 & 0.033 & $0.019-0.057$ & \\
\hline \multirow{2}{*}{$\begin{array}{l}\text { surgical approach; } \\
\text { thoracoscopy }\end{array}$} & yes & 2 & 180 & 0.011 & $0.003-0.044$ & $p=0.081$ \\
\hline & no & 70 & 2457 & 0.028 & $0.023-0.036$ & \\
\hline \multirow[t]{2}{*}{ postope. chemo } & yes & 40 & 1547 & 0.026 & $0.019-0.035$ & $p=0.317$ \\
\hline & no & 30 & 1035 & 0.029 & $0.020-0.041$ & \\
\hline \multirow[t]{2}{*}{ posope. rad } & yes & 39 & 1302 & 0.03 & $0.022-0.041$ & $p=0.190$ \\
\hline & no & 31 & 1280 & 0.024 & $0.017-0.034$ & \\
\hline pathological stage & 0 & 6 & 316 & 0.019 & $0.009-0.042$ & \\
\hline \multirow[t]{4}{*}{ (JSED, 9th edition) } & 1 & 27 & 852 & 0.032 & $0.022-0.046$ & \\
\hline & 2 & 4 & 230 & 0.017 & $0.007-0.046$ & \\
\hline & 3 & 22 & 787 & 0.028 & $0.018-0.042$ & \\
\hline & $4 \mathrm{~A} / 4 \mathrm{~B}$ & 10 & 406 & 0.025 & $0.013-0.046$ & \\
\hline
\end{tabular}

multivariate survival analyses. Data were collected on ten conditions for univariate analyses, as listed in Table 2, such as sex (male versus female), past illness that may have affected peri-operative course such as cardiopulmonary diseases or a history of gastrectomy, liver functions using Indocyanine Green clearance test-residual ratio in fifteen minutes(ICGR15), electrocardiogram (ECG), surgical approaches-transthoracic (open surgery) or not, or thoracoscopic esophagectomy or not-, and whether or not accompanying chemo- and/or radio-therapy (CRT/RT) was conducted. Twelve conditions for multivariate analyses such as sex, liver function (ICGR15), ECG, accompanying CRT/RT, as well as age, weight and height at hospitalization, pathological staging of diseases according to Japanese Society for 
Table 3. Hazard ratios and 95\% confidence intervals for non-esophageal cancer death among esophageal cancer patients.

\begin{tabular}{ccccc}
\hline factor & hazard ratio & $95 \%$ Conf. Interval* & $p$-value & \\
\hline sex & 2.42 & $0.82-7.18$ & 0.11 & male, against female \\
age at operation & 1.05 & $1.01-1.08$ & 0.01 & per year \\
ICG abnormality & 0.97 & $0.57-1.63$ & 0.9 & yes, against no abnormality \\
ECG abnormality & 1.66 & $0.97-2.82$ & 0.06 & yes, against no abnormality \\
weight at hospitalization & 0.95 & $0.91-0.98$ & 0.01 & per kirogram \\
height at hospitalization & 1.06 & $1.01-1.11$ & 0.02 & per cm \\
preoperative radiation & 3.7 & $1.29-10.61$ & 0.02 & yes, against no radiation \\
preoperative chemotherapy & 0.17 & $0.02-1.47$ & 0.11 & yes, against no chemotherapy \\
transthoracic approach & 0.77 & $0.36-1.65$ & 0.5 & yes, against non-transthoracic \\
p-stage I & 1.89 & $0.66-5.41$ & 0.24 & against p-stage0 \\
p-stage II & 1.29 & $0.30-5.61$ & 0.73 & against p-stage0 \\
p-stage III & 2.39 & $0.78-7.31$ & 0.13 & against p-stage0 \\
p-stage IVA or IVB & 1.45 & $0.42-5.08$ & 0.56 & against p-stage0 \\
postoperative radiation & 1.34 & $0.66-2.70$ & 0.42 & yes, against no radiation \\
postoperative chemotherapy & 0.65 & $0.31-1.37$ & 0.26 & yes, against no chemotherapy \\
\hline *95\% confidence interval & & & &
\end{tabular}

Esophageal Diseases, the Guidelines for clinical and pathologic studies on carcinoma of the esophagus, ninth edition [8], and the surgical approach-whether transthoracic, transhiatal, thoracoscopic esophagectomy, or upper esophagectomy with pharyngolaryngectomy with upper mediastinal lymphadenectomy under median sternotomy was conducted. The details of the surgery are as follows: transthoracic esophagectomy was performed under right thoracotomy, basically through the fifth intercostal space, with a separate laparotomy and occasional cervical lymphadenectomy [14].

Thoracoscopic esophagectomies have been performed since 1995, and the details of the procedure have basically remained the same since the first case as described by Akaishi et al. [1]; in brief, all intrathoracic maneuvers were carried out through six placed trocars, followed by a separate laparotomy and cervical lymphadenectomy. Transhiatal esophagectomies were carried out through a laparotomy and cervical incision, and a mediastinoscope has been used since 2000 [20]. In certain cases of cervical esophageal carcinoma, upper mediastinal lymphadenectomy with median sternotomy was carried out because of the presence of lymphnodes suspected of metastases in the mediastinum or because the tumor was located at the cervico-thoracic junction $[6,16]$.

Radiation therapy (RT) or chemoradiation therapy (CRT) was generally performed by radiologists or oncologists in Tohoku University Hospital. Forty-five patients underwent preoperative RT/CRT; doses of radiation of no more than $40 \mathrm{~Gy}$, between $41 \mathrm{~Gy}$ and $49 \mathrm{~Gy}$, or of no less than $50 \mathrm{~Gy}$ were administered to 22, 4, or 19 patients, respectively. The doses of radiation were determined by radiologists; the primary lesion and the regional lymphnodes were generally irradiated with up to $40 \mathrm{~Gy}$ using anterior-posterior opposed fields with conventional fractionation (1.5-2 Gy/day, 5 times/week), then the beam direction was changed to avoid the spinal cord [12,13]. After finishing 40 to 70 Gyirradiation, the tumor was evaluated by CT exam, barium esophagogram and endoscopy, and the patients were transferred to the Department of Surgery if the tumors were surgically removable. As a majority of preoperatively irradiated cases were advanced cancer or had a co-morbidity such as liver dysfunction, we assessed the pathological stages of surgically removed tumors and preoperative general conditions by multivariate analyses in the later part of this study.

As for the accompanying chemotherapy, cisplatin (CDDP) and 5-FU were usually used as preoperative chemotherapy, but several regimens were used as preoperative chemotherapy by a number of radiologists or oncologists. Between 1987 and 1993, double-blinded random assignment was used to assign patients to groups receiving either radiochemotherapy or aggressive chemotherapy alone in a previous study as the postoperative adjuvant therapy [14]; postoperative radiochemotherapy consisted of $40 \mathrm{~Gy}$ of T-shaped irradiation and a combination of 5-FU, vindesine, and tumor necrosis factor, and the postoperative aggressive chemotherapy was characterized by a combination of pepleomycin, adriamycin and mitomycin, as described elsewhere [15].

Our department policy is for life-long follow-up, which means clinic visits every four months for the first two years, every six months for the next three years, and at least every year for the following years, usually, at out-patient clinics of Tohoku University Hospital or other affiliated hospitals. Further investigations such as CT and endoscopy were performed at those visits. Additionally, all survivors were mailed and scored by questionnaires once a year. Survival time was measured from the date of surgery to the date of the last ascertainment of survival status or until October 31, 2003 , the end of this study. The cause of death was identified from pathological reports if they had autopsies, hospital case-notes or hospital reports from affiliated hospitals. For multivariate analysis, to exclude the possible influence by a sampling bias, the Cox proportional hazards regression model was employed. Hazard ratios of potential risk factors of death were estimated by the conditional maximum likelihood method, taking into account for other factors in the 
model. Statistical analysis was performed using the statistical software of STATA $[8,18]$. All statistical tests were twosided and significance was defined as $p<0.05$.

\section{Cox proportional hazards model}

Cox proportional hazards model is a regression method described by D. R. Cox in 1972 for modeling survival times [5]. It is called proportional hazards model because it estimates the ratio of the risks (hazard ratio or relative hazard); the model assumes that the underlying hazard rate (rather than survival time) is a function of the independent variables and consistent over time (proportionality assumption, i.e. the survival functions of the groups are approximately parallel). As in any regression model, there are multiple predictor variables (such as prognostic markers whose individual contribution to the outcome is being assessed in the presence of the others) and the outcome variable (e.g., whether the patients died during follow-up, etc.). Therefore, Cox regression can allow for differences in the baseline characteristics of the groups that are being compared, whether in a randomized or a nonrandomized trial.

$$
h(t)=\left[h_{0}(t)\right] e^{\left(b_{1} X_{1}+b_{2} X_{2}+\cdots b_{k} X_{k}\right)}
$$

This model can also be expressed as:

Relative Hazard

$$
\begin{aligned}
& \left(\frac{h(t)}{h_{0}(t)}\right)=e^{\left(b_{1} X_{1}+b_{2} X_{2}+\cdots b_{k} X_{k}\right)} \\
& h(t)=\text { the hazard function at time } t \\
& h_{0}(t)=\text { the baseline hazard or hazard for an individual when the value of all the independent } \\
& \quad \text { variables equal zero. }
\end{aligned}
$$

\section{Results}

\section{Patient characteristics and survival rate}

The mean age of 523 patients undergoing esophagectomy for esophageal cancer was 64.0 years (standard deviation: 8.7 years) and $449(85.9 \%)$ were men; 441 (84.3\%) underwent transthoracic esophagectomy, while 40 (7.6\%) underwent thoracoscopic esophagectomy, 39 (7.5\%) underwent transhiatal esophagectomy and three $(0.6 \%)$ underwent upper esophagectomy with pharyngolaryngectomy with upper mediastinal lymphadenectomy through median sternotomy (Table 1). Two hundred and seven out of $523(39.6 \%)$ patients died from esophageal carcinoma, and 72 $(13.8 \%)$ died from causes other than esophageal cancer as of the end of October 2003. Five patients $(0.96 \%)$ died within 30 days postoperatively. The median survival time for all causes of death was 6.0 years. The 1-, 3-, and 5-year over-all survival rates were $80.7 \%$ (95\% CI: 0.77-0.84), 59.4\% (95\% CI: 0.55-0.64), and 53.4\% (95\% CI: 0.49-0.58), respectively. On the other hand, for the cause-specific esophageal cancer, the 1-, 3-, and 5-year survivals were $83.0 \%$ (95\% CI: 0.795-0.860), 64.3\% (95\% CI: 0.599-0.684), and 59.4\% (95\% CI: 0.549-0.637), respectively (Fig. 1). The overall survival curve was quite similar to that of the cause-specific survival curve within five years, postoperatively, although the mortality due to non-esophageal cancer causes rather seemed to have influenced the overall survival after

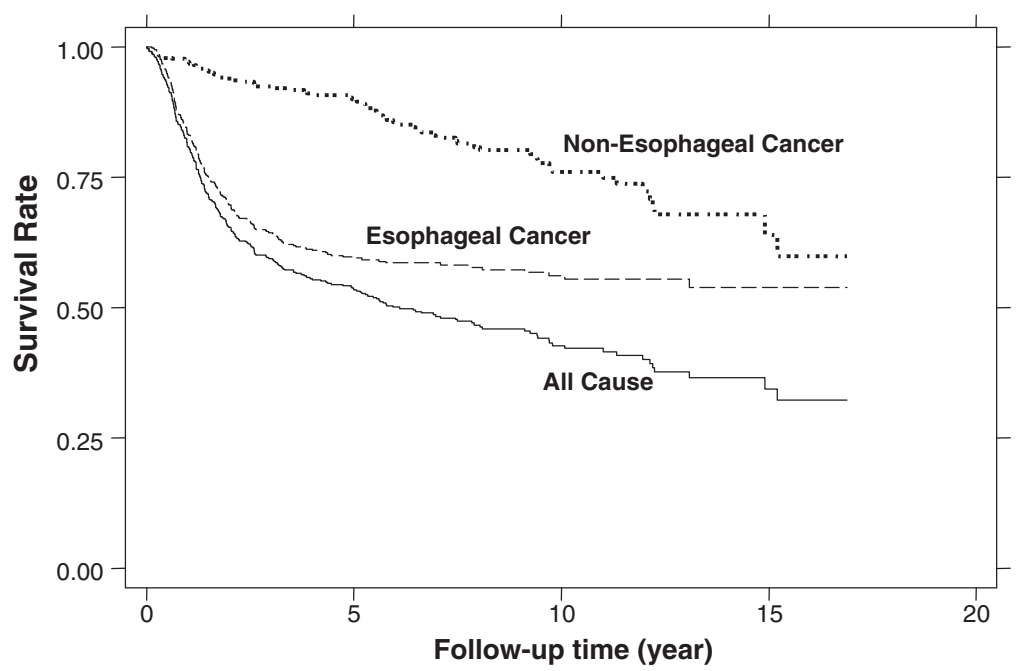

Fig. 1. Kaplan-Meier estimates of survival rates of 523 cases examined in this study by cause of death. The median survival time for overall causes of death was 6.0 years. Mortality due to non-esophageal cancer causes is indicated by a dotted line, and the mortality from esophageal cancer is indicated by a broken line. 
five years postoperatively, as these two survival curves were compared. This result implies that a more detailed evaluation of plausible risk factors potentially influencing the mortality from other diseases in the later follow-up period may be necessary. Hence, we focused on fatal cases of non-esophageal cancer causes in this study; the total number of cases of non-esophageal cancer death was 118. Among them, the numbers of cases that died from cerebrovascular, respiratory system-related, cardiovascular, gastrointestinal system-related, or other diseases including renal failure, sepsis, postoperative multiple organ failure, etc. were five, twenty three, eleven, ten, and seven, respectively. Cases of sudden death without any further available details were six. We examined these sixty-two cases as the group of mortality from causes other than esophageal cancer, as we excluded the other fifty-six cases because they were dead from other cancers, accident, or unknown reasons.

\section{Univariate analysis}

Univariate analysis revealed that sex (male against female) $(p=0.005)$, abnormality on ECG $(p=0.012)$, and the presence of preoperative radiation $(p=0.016)$ significantly increased the incidence of mortality from reasons other than esophageal cancer; on the other hand, surgical approach such as thoracoscopic approach almost significantly $(p=0.081)$ decreased the incidence rate of non-esophageal cancer death (Table 2).

\section{Multivariate analysis}

Multivariate analysis revealed that the presence of preoperative radiation, age at operation, and height at hospitalization increased and the weight at hospitalization decreased the risk for mortality from other diseases with statistical significance $(p<0.05)$. Hazard ratios were 3.70 (95\% CI: 1.29-10.61) for preoperative radiation, 1.05 (95\% CI: 1.01-1.09) for one year of age, 1.06 (95\% CI: 1.01-1.11) for one cm of height and 0.95 (95\% CI: 0.91-0.98) for one $\mathrm{kg}$ of weight, respectively (Table 3). On the other hand, neither pre/post-operative chemotherapy nor postoperative radiotherapy significantly increased the risk for mortality from non-cancer causes with 0.17 (95\% CI: 0.02-1.47), 0.65 (95\% CI: 0.31-1.37) and 1.34 (95\% CI: 0.66-2.70) in hazard ratios, respectively. In this study, neither an abnormality in preoperative ICG tests nor in ECGs was shown to be a significant risk factor for mortality from non-cancer causes, as their hazard ratios were 0.97 (95\% CI: 0.57-1.63) and 1.66 (95\% CI: 0.97-2.82), respectively (Table 3).

\section{Survival of esophageal cancer patients with or without preoperative radiation}

As shown in the multivariate analysis, the presence of preoperative radiation significantly increased the risk for mortality from diseases other than esophageal cancer. We show here the survival curves of postoperative esophageal cancer patients with or without preoperative radiation using an estimated baseline survivor function of the fitted Cox proportional model with the values of the other factors described in this study equal to their means, including age, several preoperative organ functions, and pathological stages (Fig. 2); the result clearly indicated that preoperative radiation significantly increased the mortality due to diseases other than esophageal cancer. Also, cases with preoperative radiotherapy were accompanied by significantly higher mortality even in the later follow-up period.

\section{Discussion}

Many clinical trials have been made to evaluate the prognosis of esophageal cancer patients by newly developed

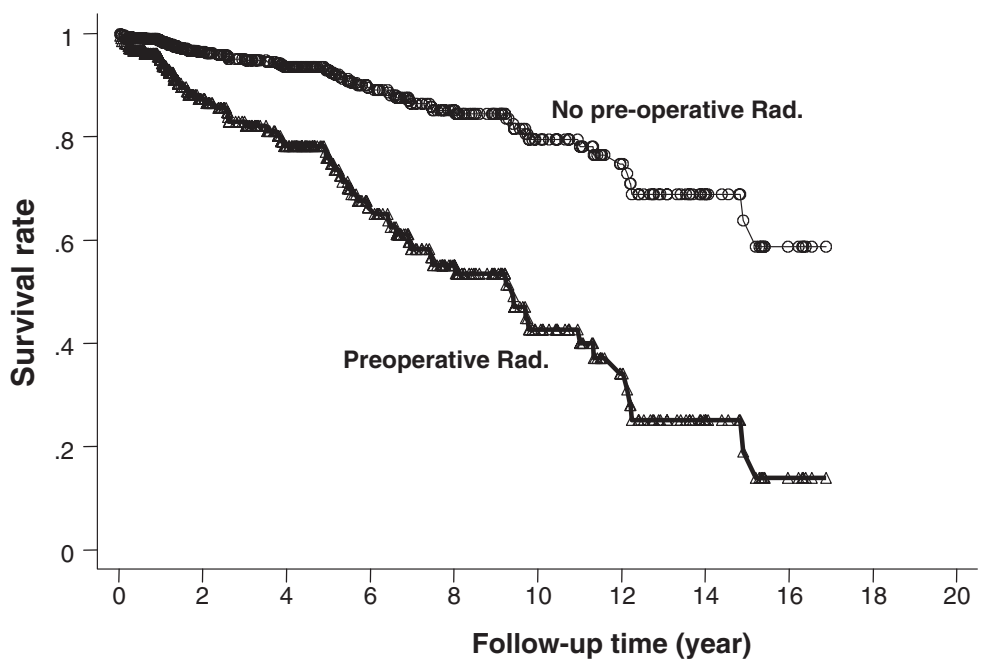

Fig. 2. The survival curves of postoperative esophageal cancer patients with or without preoperative radiation using an estimated baseline survivor function of the fitted Cox proportional model with the values of the other factors examined in this study equal to their means. The line with an open circle and the broad line with the triangle represent the postoperative survival curves of cases without preceding radiation and cases with preceding radiation, respectively. 
treatments, and quite a few reports described that multi-modal therapy improved the prognosis of esophageal cancer patients. While little doubt remained that multi-modal therapy improved the mortality from the recurrence of esophageal cancer, it would also be important to determine the influence of the therapy modality on postoperative morbidity if the cases are accompanied by a high incidence of diseases other than esophageal cancer in the later followup period.

First, we analyzed potential risk factors by evaluating the incidence of mortality from non-esophageal cancer causes, and found that sex-male against female-, abnormality on ECG, and the presence of preoperative radiation were significant risk factors. Conversely, the thoracoscoic procedure decreased the incidence rate of non-esophageal cancer death although the difference was not statistically significant $(p=0.081)$. It may be suggested that cases with preoperative radiation may have had a greater risk for a poor postoperative course from the co-morbidity of other diseases or because the tumor was so advanced that it may have caused operative difficulties. The fact that the incidence of non-esophageal cancer death increased even in the later follow-up period suggests that this would be unlikely, although it may be necessary to fit other factors equal to their means. Hence, we next employed multivariate analysis of the preoperative risk factors, tumor evaluation by pathological staging, sex, as well as whether preoperative RT/CRT was conducted, to evaluate whether these potential risk factors were independent of each other. Among fifteen factors, only four were significantly associated with the postoperative mortality from diseases other than esophageal cancer: the age at surgery, the presence of preoperative radiation, and the weight and height at hospitalization.

Advanced age at surgery naturally increases the risk for other diseases, probably because of the increasing incidence of age-related illnesses rather than because of surgical complications. That the weight at the time of hospitalization was a risk factor is thought to reflect the fact that some cases with extreme weight loss due to long-term inability to take food orally intake may have had a relatively higher risk for other diseases after surgery than cases without weight loss. However, further investigation may be necessary to determine the mechanism underlying the relationship between postoperative non-esophageal cancer death and the age or weight. The result concerning height at hospitalization is consistent with the fact that shorter people generally have greater longevity than taller people as described by Samaras et al. [18], and may support the accuracy of our multivariate analysis.

Preoperative radiation therapy had one of the most significant hazards of non-cancerous death compared with no radiation therapy $(3.70,95 \%$ CI: $1.29-10.61)$ in the present study. Several reports described that the risks for postoperative pneumonia and enteritis increased as a result of preoperative radiation or chemoradiation [3,11,22]. Furthermore, in the present study we were able to show clearly that, among potential factors examined, preoperative radiation most significantly increased the risk of mortality caused by other diseases. We also showed the fact that the mortality from other causes than esophageal cancer increased even in the later follow-up period, as the other potential factors such as preoperative liver or cardiac function, age, pathological stage of tumor, etc. were adjusted as equal to their means. This fact would suggest that patients who underwent esophagectomy after radiation therapy increased certain damages not only from postoperative surgical complications but also from currently unknown causes that affected even the later follow-up period. This was shown in the survival analyses of the postoperative esophageal cancer patients with or without preoperative radiation using an estimated baseline survivor function of the fitted Cox proportional model with the values of the other factors such as pathological stages of tumor, preoperative general conditions, age, sex, etc. examined in this study equal to their means (Fig. 2). Therefore, it should be quite important to investigate further the relationship between preoperative radiation and later causes of death. We found that postoperative restrictive changes of the lung were more obvious in cases with preoperative radiation therapy (data not shown) and infer that this may have contributed to the increased risk for morbidity from other diseases in the follow-up period. We speculate that the restrictive changes might have been aggravated by pleuritis or intrathoracic lung adhesions from the thoracotomy after radiation as radiation and thoracotomy themselves obviously provide an opportunity for the development of pleuritis and intrathoracic adhesions. However, the details underlying this relationship are still unclear and further investigation is necessary. Physicians and surgeons should be encouraged to develop new approaches such as thoracoscopic esophagectomy to cases with preoperative radiation therapy towards decreasing the chance of restrictive damages in the lung, since the decrease in the incidence of non-esophageal cancer death with the thoracoscopic procedure, although not statistically significant, may have resulted from a decrease in the development of pleuritis and intrathoracic adhesions (paper in preparation).

Many studies have been conducted to shed light on advances in cancer-specific survival so far. However, the results we show here underscore the necessity of this kind of study concerning morbidity in the entire follow-up period to improve the overall survival of esophageal carcinoma patients.

\section{Acknowledgement}

The authors are grateful to Dr. Kenji Nemoto, Tohoku University Department of Radiology, for his helpful advice, especially concerning the details of accompanying radiotherapies. 


\section{REFERENCES}

[1] Akaishi, T., Kaneda, I., Higuchi, N., Kuriya, Y., Kuramoto, J., Toyoda, T., and Wakabayashi, A., "Thoracoscopic en bloc total esophagectomy with radical mediastinal lymphadenectomy," J. Thorac. Cardiovasc. Surg., 112: 1533-1540 (1996).

[2] Arnott, S. J., Duncan, W., Gignoux, M., Girling, D. J., Hansen, H. S., Launois, B., Nygaard, K., Parmar, M. K., Roussel, A., Spiliopoulos, G., Stewart, L. A., Tierney, J. F., Mei, W., and Rugang, Z., "Preoperative radiotherapy in esophageal carcinoma: A meta-analysis using individual patient data (esophageal cancer collaborative group)," Int. J. Radiat. Oncol., Biol., Phys., 41: 579-583 (1998).

[3] Avendano, C. E., Flume, P. A., Silvestri, G. A., King, L. B., and Reed, C. E., "Pulmonary complications after esophagectomy," Ann. Thorac. Surg., 73: 922-926 (2002).

[4] Bosset, J. F., Gignoux, M., Triboulet, J. P., Tiret, E., Mantion, G., Elias, D., Lozach, P., Ollier, J. C., Pavy, J. J., Mercier, M., and Sahmoud, T., "Chemoradiotherapy followed by surgery compared with surgery alone in squamous cell cancer of the esophagus," N. Engl. J. Med., 337: 161-167 (1997).

[5] Cox, D. R., "Regression models with life tables," J. of the Royal Statistical Society, 34: 187-220 (1972).

[6] Fujita, H., Kakegawa, T., Inoue, Y., Yamana, H., Shirouzu, G., Minami, T., and Tai, Y., "Upper esophagectomy with pharyngolaryngectomy for esophageal carcinoma at the cervicothoracic junction," Jpn. J. Surg., 21: 650-654 (1991).

[7] Gignoux, M., Roussel, A., Paillot, B., Gillet, M., Schlag, P., Favre, J. P., Dalesio, O., Buyse, M., and Duez, N., "The value of pre-operative radiotherapy for carcinoma of the oesophagus," World J. Surg., 153: 426-432 (1987).

[8] Japanese Society for Esophageal Diseases. Guidelines for clinical and pathogic studies on carcinoma of the esophagus, ninth edition. Esophagus, 1, 61-88 (2004).

[9] Kalbfleisch, J. D., and Prentice, R. L., The Statistical Analysis of Failure Time Data, Jon Wiley \& Sons, Inc., Hoboken, New Jersey (2002).

[10] Le Prise, E., Etienne, P. L., Meunier, B., Maddern, G., Ben Hassel, M., Gedouin, D., Boutin, D., Campion, J. P., and Launois, B., "A randomized study of chemotherapy, radiation therapy, and surgery versus surgery for localized squamous cell carcinoma of the esophagus," Cancer, 73: 1779-1784 (1994).

[11] Lee, H. K., Vaporciyan, A. A., Cox, J. D., Tucker, S. L., Putnam, J. B., Jr., Ajani, J. A., Liao, Z., Swisher, S. G., Roth, J. A., Smythe, W. R., Walsh, G. L., Mohan, R., Liu, H. H., Mooring, D., and Komaki, R., "Postoperative pulmonary complications after preoperative chemoradiation for esophageal carcinoma: correlation with pulmonary dose-volume histogram parameters," Int. J. Radiat. Oncol., Biol., Phys., 57: 1317-1322 (2003).

[12] Nemoto, K., Matsumoto, Y., Yamakawa, M., Jo, S., Ito, Y., Oguchi, M., Kokubo, N., Nishimura, Y., Yamada, S., and Okawa, T., "Treatment of superficial esophageal cancer by external radiation therapy alone: rescue of a multi-institutional experience," Int. J. Radiat. Oncol., Biol., Phys., 46: 921-925 (2000).

[13] Nemoto, K., Takai, Y., Ogawa, Y., Kakuto, Y., Ariga, H., Matsushita, H., Wada, H., and Yamada, S., "Fatal hemorrhage in irradiated esophageal cancer patients," Acuta Oncologia, 34: 259-262 (1998).

[14] Nishihira, T., Hirayama, K., and Mori, S., "A prospective randomized trial of extended cervical and superior mediastinal lymphadenectomy for carcinoma of the thoracic esophagus," Am. J. Surg., 175: 47-51 (1998).

[15] Nishihira, T., Hirayama, K., Shineha, R., Akaishi, T., Takano, R., Kitamura, M., and Mori, S., "Postoperative adjuvant therapies for cancer of the thoracic esophagus on the basis of the extent of node metastasis," Chirurgia Toracica, 43: 4-12 (1990).

[16] Orringer, M. B., "Partial median sternotomy: anterior approach to the upper thoracic esophagus," J. Thorac. Cardiovasc. Surg., 87: 124-129 (1984).

[17] Parkin, D. M., Bray, F., Ferlay, J., and Pisani, P., "Estimating the world cancer burden: Globocan 2000," Int. J. Cancer, 94: 153-156 (2001).

[18] Samaras, T. T., Storms, L. H., and Elrick, H., "Longevity, mortality and body weight," Aging Research Reviews, 4: 673-691 (2002).

[19] StataCorp. Sata Statistical Software: release 7.0. College Station, TX: Sata Corporation (2001).

[20] Tangoku, A., Hayashi, H., Kanamura, S., Yoshino, S., Abe, T., Yoshimoto, Y., Morioka, T., and Oka, M., "Lymph node metastases identified with mediastinoscopy in a patient with superficial carcinoma of the esophagus," Surg. Endosc., 14: 595 (2000).

[21] Urba, S., Orringer, M., Turrisi, A., Iannettoni, M., Forastiere, A., and Strawderman, M., "Randomized trial of preoperative chemoradiation versus surgery alone in patients with locoregional esophageal carcinoma," J. Clin. Oncol., 19: 305-313 (2001).

[22] Urba, S. G., Orringer, M. B., Perez-Tamayo, C., Bromberg, J., and Forastiere, A., "Concurrent preoperative chemotherapy and radiation therapy in localized esophageal adenocarcinoma," Cancer, 69: 285-291 (1992).

[23] Walsh, T. N., Noonan, N., Hollywood, D., Kelly, A., Keeling, N., and Hennessy, T. P., "A comparison of multimodal therapy and surgery for esophageal adenocarcinoma," N. Engl. J. Med., 335: 462-467 (1996). 